\title{
Prostate cancer: any room left for immunotherapies?
}

\author{
Constantin N Baxevanis*,1, Sotirios P Fortis ${ }^{1}$ \& Sonia A Perez ${ }^{1}$ \\ ${ }^{1}$ Cancer Immunology \& Immunotherapy Center, Saint Savas Cancer Hospital, Athens, Greece \\ *Author for correspondence: Tel..: +30 210640 9624; baxevanis@ciic.gr
}

First draft submitted: 11 October 2018; Accepted for publication: 29 October 2018; Published online: 11 January 2019

Keywords: $\mathrm{CD}^{+} \mathrm{T}$ cells $\bullet$ endogenous immunity $\bullet$ immune checkpoint blockade $\bullet \mathrm{MHC}$ class I $\bullet$ mutational load $\bullet$ PD-1/PD-L1 • prostate cancer

\section{Endogenous antitumor immunity is an integral part of the treatment of cancer patients}

The field of cancer therapeutics has been greatly advanced via remarkable progresses in cancer immunology and immunotherapy. The 'immunoediting theory' provided convincing preclinical evidence in support of the notion that endogenous antitumor immunity is shaped by the immune system and determines tumor evolution [1]. Accumulating clinical evidence suggested that anticancer therapies prior to induce effective and long lasting clinical responses should reinstate the endogenous immunity [1]. Our knowledge about regulation of endogenous antitumor immunity has been greatly enhanced through the discovery of immune checkpoint inhibitors (e.g., CTLA-4, PD-1) followed by the generation of monoclonal antibodies targeting such inhibitory receptors and their ligands. Immunotherapy based on immune checkpoint inhibition has uncovered the indispensable role of the endogenous immunity for controlling tumor growth. By interrupting the interaction between the checkpoint inhibitory receptors and their cognate ligands, a robust and sustainable antitumor adaptive immune response can be elicited. These findings strongly suggest that patients' immune system has the capacity to specifically react against their autologous tumors upon recognition of tumor-specific antigens. The precise immune pathways that dictate tumor control are yet not thoroughly characterized, although several markers, including PD-L1 expression, mutational burden (mostly in tumors with microsatellite instability and somatic mutation-associated neoantigens) as well as immune infiltrates have been evaluated in certain tumor types [2]. Cumulative data highlighted these determinants as predictive biomarkers for immunotherapy efficacy, and potentially crucial targets for targeted immune therapy interventions. The success of checkpoint inhibition-based therapies in cancer patients with high immunoscores or with PD-L1-expressing tumors underlines the notion that tumor-specific $\mathrm{T}$ cell responses pre-exist in some patients and are kept under tight control via immune modulatory mechanisms. Most patients who have endogenous tumor responses maintain durable disease control upon immune checkpoint inhibition, although $30-40 \%$ of them relapse. The cellular and molecular mechanisms of acquired resistance to checkpoint blockade therapy are currently only poorly understood, but their description will allow for designing effective combination immunotherapy approaches.

\section{The CD8 ${ }^{+}$T cell / PD-1-PD-L1 / HLA-I / T cell inflamed tumors axis for successful immunotherapies}

Endogenous antitumor immunity comprising mostly $\mathrm{CD} 8^{+} \mathrm{T}$ cell immune infiltrates within the tumor microenvironment is of paramount importance as a significant prognosticator for patients' clinical outcome, but also for predicting responses to therapeutic treatments and for helping to discover new therapeutic modalities. Tumors overpopulated by cytotoxic $\mathrm{CD} 8^{+} \mathrm{T}$ cells are characterized by a series of traits including: increased immunogenicity through the production of immunogenic endogenous peptides; microsatellite instability with high mutational load; and high PD-1/PD-L1 expression [2]. Such immunogenic tumors usually will respond to immunotherapies mostly in the form of immune checkpoint (anti-PD-1/anti-PD-L1) blockade and even to targeted therapies or conventional therapies that can activate $\mathrm{CD} 8^{+} \mathrm{T}$ cells through various mechanisms including immunogenic cell death or reversing tumor-induced suppression. Therefore, cytotoxic $\mathrm{CD} 8^{+} \mathrm{T}$ cells are the forefront of the immune attack against the tumor making the effective killing of tumor cells by activated $\mathrm{CD} 8^{+} \mathrm{T}$ cells the ultimate goal 
to achieve during cancer immunotherapies. The $\mathrm{CD} 8^{+} \mathrm{T}$ cell-dependent killing of cancer cells requires efficient presentation of tumor antigens by human leukocyte antigen class I (HLA-I) molecules. The antitumor activity of immune checkpoint blockade depends on $\mathrm{CD}^{+} \mathrm{T}$ cell and HLA class I-restricted immune activity with distinct HLA supertypes being associated with extended or poor survival [3]. Moreover, HLA class II genes were found to be differentially expressed between responders versus nonresponders during immune checkpoint therapies [4], which are in line with the requirement of HLA-II dependent $\mathrm{T}$ cell help for optimal activation of $\mathrm{CD} 8^{+} \mathrm{T}$ cell cytotoxicity. However, tumor cells evade immune surveillance by $\mathrm{CD} 8^{+} \mathrm{T}$ cells variously, including defective IFN $\gamma$ signaling and downregulation of their HLA-I molecules. There is also a direct correlation between intratumoral levels of tumor HLA-I expression and the degree of $T$ cell infiltrates [5]. Hence, immunotherapeutic modalities in cancer should aim at restoring these deficiencies, thereby enhancing tumor recognition and killing by $\mathrm{CD} 8^{+}$ T cells. Prostate tumors are characterized by high levels of HLA-I downregulation or loss [6] resembling with immune surveillance escaping non- $\mathrm{T}$ cell-inflamed tumors characterized by weak $\mathrm{CD} 8^{+} \mathrm{T}$ cell infiltration and low PD-1/PD-L1 expression. Combination therapeutic modalities including immunotherapies and targeted therapies may be utilized to induce clinical benefit for patients with the non-T cell-'cold' tumors. The current trend of novel strategies (e.g., immune checkpoint blockade and chimeric antigen receptor $T$ cell therapy) to induce or promote antitumor $\mathrm{T}$ cell immunity in the setting of the non-T cell-inflamed tumors ultimately may result in functional renaissance of immune mechanisms advancing endogenous $T$ cell responses. Reactivation of endogenous adaptive immunity in tumors already infiltrated by immune lymphocytes may result in the mutual activation of both innate and adaptive immunity followed by the production of cytokines including interferons with induction of suppressor circuits. Such a negative feedback regulation of antitumor immunity will induce adaptive immune resistance via IDO release by and expression of PD-L1 on the tumor cells [7]. Therefore, when applying immunotherapies or targeted therapies, it is important to combine those with agents blocking pathways leading to immune resistance to achieve durable clinical responses. Recruitment of tumor-specific T lymphocytes 'cold' tumors may also generate effective antitumor immunity provided that such tumors will harbor mutations coding for immunogenic neoantigens [7]. Also, in this case combinatorial treatments will be needed to inhibit induction of immune resistance mechanisms by the tumor cells [8]. These data support the notion that non-T cell inflamed tumors do indeed express candidate antigens and so therapeutic vaccines targeting such neoantigens could also induce tumor infiltration by neoantigen-specific $\mathrm{T}$ cells. This would represent an additional intervention aiming at initiating a $\mathrm{T}$ cell-inflamed tumor microenvironment de novo.

\section{The paradox in prostate cancer immunotherapy}

Prostate cancer presents a confounding issue regarding its immunogenicity. A cellular therapeutic cancer vaccine (sipuleucel-T) demonstrated a significantly extended overall survival (OS) in a Phase III trial, and there are many reports on clinical benefits from other smaller studies based on therapeutic vaccines which indicate prostate tumors could be immune responsive, and therefore amenable to immunomodulation in combination trials [9]. In contrast, survival benefits from clinical trials with immune checkpoint inhibitors including PD-1 and PD-L1 targeting, yielded modest clinical results [9]. Regarding ipiliumumab, results of a Phase I/II study, chemotherapy-naive or chemotherapy-pretreated patients with metastatic CRPC (mCRPC) showed antitumor activity in this clinical setting, with durable prostate-specific antigen responses independent of prior chemotherapy [10]. However, when tested in Phase III trials, ipilimumab did not improve OS in mCRPC patients although detectable increases in progression-free survival and prostate-specific antigen response rates suggested antitumor activity at least in a small cohort of patients [10]. Markedly, when ipilimumab was applied plus androgen-deprivation therapy before surgery in patients with localized prostate cancer, significant increases in PD-L1 as well as in tumor-infiltrating immune cells, including $\mathrm{CD}^{+}, \mathrm{CD}^{+}, \mathrm{ICOS}^{+}, \mathrm{CD} 45 \mathrm{RO}^{+}$, granzyme- ${ }^{+}$and $\mathrm{CD} 8^{+}$cells could be detected by immunohistochemistry in post-treatment tumors which were not seen in the control group treated with androgen-deprivation therapy alone [11]. More important, VISTA, another immune checkpoint, was also increased in the tumor microenvironment. Thus, in the tumor microenvironment, ipilimumab could promote an immune response across multiple subsets with a concomitant upregulation of PD-L1 and VISTA as adaptive immune resistance mechanisms. Recent data have suggested that sustained IFN $\gamma$ signaling in the tumor microenvironment contributes not only to PD-L1 expression but also to PD-L1 independent adaptive immune resistance via the expression of additional immune checkpoints [12]. Therefore, the expression of multiple immune checkpoints as a result of a massive intratumoral immune response may pose a significant barrier to clinical efficacy using immune checkpoint inhibition. This underlines the complexity of an induced adaptive immune response against the tumor 
with a variety of immune suppressor mechanisms, which need to be counteracted via combination therapies. Based on this and from a broader perspective of view, immunotherapies must employ modalities that promote intratumor immune cell infiltration and, at the same time, protect these cells from being suppressed or exhausted when they reach the various tumor compartments. Therefore, it will be important to evaluate the effect of immunotherapies within the tumor microenvironment, and to identify potentially relevant regulatory or resistance mechanisms. The tumor microenvironment could present an anatomical site where, dependent on the context, various successive mechanisms of immune resistance will result in sequential upregulation of multiple immune checkpoints. This could propose the existence of heterogeneous immunological milieu within each single patient at the various metastatic sites. Such a pluralism of immune checkpoints could then provide a serious obstacle for achieving clinical benefits, even if the underlying immunotherapy can increase lymphocyte infiltration and drive increased PD-L1 expression intratumorally.

\section{Current treatments for prostate cancer: is there room left for immunotherapies?}

Recent advances in prostate cancer biology have significantly moved forward the field of therapy in this particular type of cancer. Drug developments suppressing androgen biosynthesis and blocking the androgen receptor used in combination have remarkably improved treatment options in patients with advanced disease [13]. These clinical improvements have resulted in new guideline recommendations, and are likely to change the standard-of-care for prostate cancer. Nevertheless, and despite this progress, prostate cancer remains incurable mostly due to the lack of long-lasting clinical responses. Activating pre-existing adaptive immune memory may be necessary to provide substantial clinical benefit for patients with prostate cancer. Recent studies suggest that pre-existing immunity in the form of tumor infiltrating memory $\mathrm{CD}^{+} \mathrm{T}$ cells represent a mandatory prognostic and predictive biomarker for colorectal cancer and other types of cancer [1,2]. In addition, tumor mutation burden and expression of neoantigens are associated with tumor infiltration by immune cells and with favorable clinical response to checkpoint inhibitor treatment [2]. Evidence from several clinical trials suggests that the strength and durability of the immune response against the tumor correlates with the presence of such neoantigens [14].

However, the field is still under intense investigation given that currently no pretreatment biomarker has been validated to be included in part of the standard-of-care decision-making for anti-PD-1/PD-L1 therapy. This may derive from the fact that epigenetic modifications increase as the tumor evolves, making heterogeneous neoantigen expression more likely than clonal expression. Prostate cancer has a comparatively low proportion of tumor-specific neoantigens suggesting that it may be less likely to respond to treatments addressing immune checkpoint inhibitors [15]. On the other hand, recently published data have shown $\mathrm{T}$ cell receptor diversity within individual metastatic lesions, which may suggest that the neoantigen expression is heterogenous within the tumors meaning that different cells may express different sets of neoantigens $[16,17]$. To this end, we may hypothesize that certain checkpoints are more relevant in certain anatomic sites than in others (e.g., lymph nodes vs tumor microenvironment) which implies the existence of abundant regulatory mechanism blunting the activity of antiPD-1/PD-L1 therapies. Nonetheless, in view of the strong survival advantage provided by therapies with immune checkpoint inhibitors in different cancer types, the potential benefits of immunotherapy are also being evaluated in prostate cancer $[10,11,18]$. In addition, assessing for the expression of immunologic signals that mobilize immune lymphocytes within the tumor could apparently help to identify patients who might respond to immunotherapies in prostate cancer. To this end, we should put efforts to define selected groups of patients who will benefit from immune checkpoint inhibition. One potential biomarker conferring sensitivity to this type of immunotherapy includes PD-L1 expression. Increased PD-L1 expression has been reported in patients with aggressive primary tumors acting as independent prognostic factor of disease progression following radical prostatectomy [19] and suggesting an association of PD-L1 with evasion of primary tumors from immunosurveillance. Castrate-resistant patients, who progressed upon antiandrogen therapies, also had increased PD-L1 expression [20] which drives to the hypothesis that PD-L1 expression on prostate tumor cells might reveal a mechanism of resistance to antiandrogens independent of androgen receptor signaling. Another strategy for developing effective immunotherapies in prostate cancer would be to elucidate mechanisms for resistance to immune checkpoint inhibition, which would help us in the design of combination therapies overcoming immune evasion. For instance, primary resistance could be reversed through vaccination for inducing $\mathrm{T}$ cell infiltration in the tumor concurrent with immune checkpoint blockade for reversing suppression. Adaptive resistance could be reversed via sequential or combined treatment with immune checkpoint inhibitors (Figure 1). It will be also useful to precisely characterize the setting of treatment, considering immune therapies a part of combination with other therapies. The other aim for future studies is to 


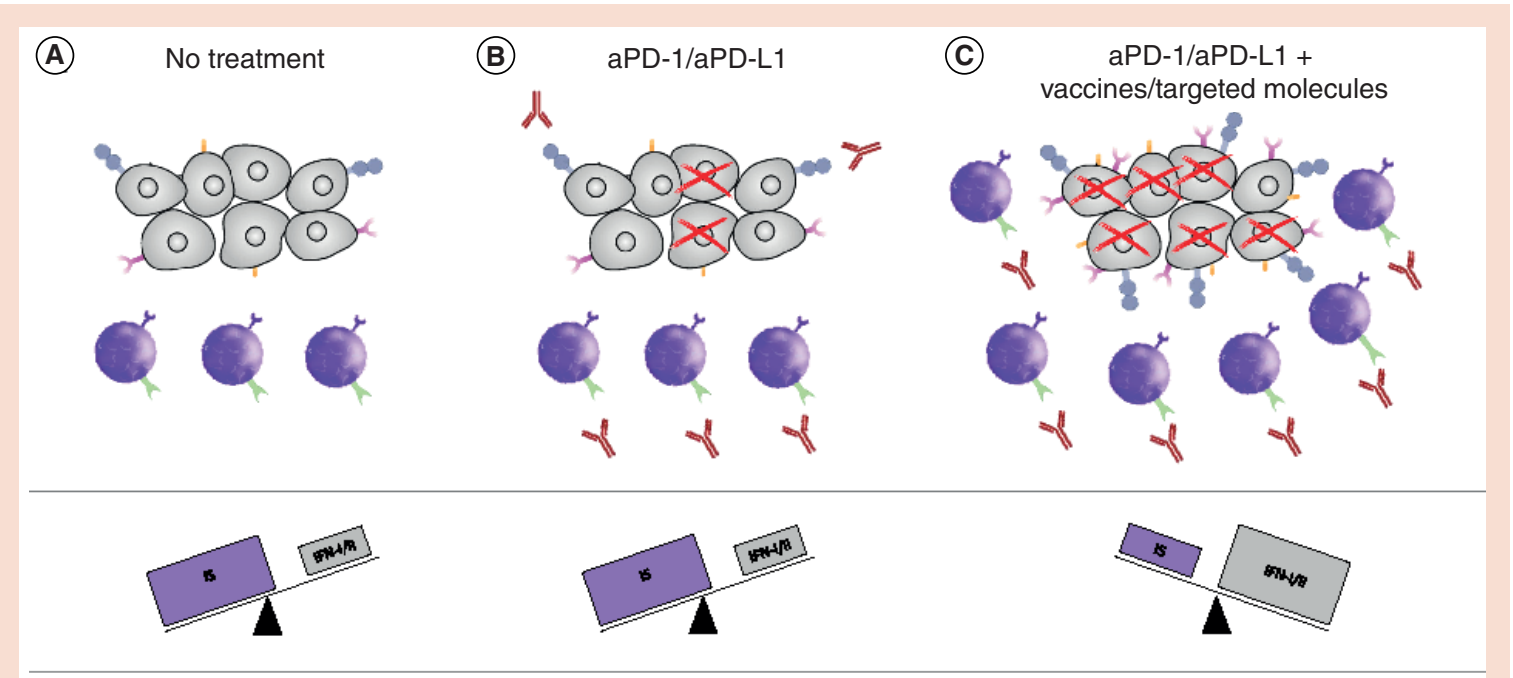

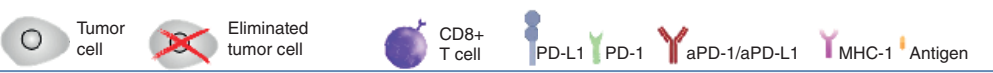

Figure 1. Combining therapeutic vaccination or targeted therapies with anti-PD-1 or anti-PD-L1 blocking antibody leads to enhanced antitumor response in prostate cancer. (A) Prostate tumors have a non-T cell inflamed phenotype that leads to immune escape through downregulation of MHC-I and prostate antigens, low PD-1/PD-L1 expression, low CD8 ${ }^{+} \mathrm{T}$ cell infiltration and an imbalanced immunosuppression (IS) (high) to type I/ll interferons (low). (B) Treatment with anti-PD-1 or anti-PD-L1 may reverse CD8 ${ }^{+}$T cell exhaustion, but still these cytotoxic cells in the hostile tumor microenvironment (high IS, low MHC-I, low proinflammatory cytokines) will not be able to exert their functional program by attacking and lysing tumor cells, with no clinical benefits for the patients. (C) Addition of therapeutic vaccine treatment (e.g., sipuleucel-T) or targeted therapies (e.g., PARP targeting agents) leads to enhanced MHC-I and prostate antigen expression, a sustained increase in CD8 ${ }^{+} \mathrm{T}$ cell infiltrates, low IS and high type I/II interferons, with a more favorable tumor microenvironment (PD-1 and PD-L1 expression are increased but are inhibited by blocking antibody) conducive to increased cancer cell death. Together, these data suggest that vaccination and targeted therapy may synergize with immune checkpoint blockade to maximize immunologic and clinical response.

IS: Immunosuppression.

find reliable biomarkers that can select patients who will most likely respond to immunotherapies. The discovery of such biomarkers could be useful for advancing personalized therapies in prostate cancer, and for better sequencing of immunotherapies with other therapeutic modalities.

\section{Perspectives in the field}

The field of therapeutics for advanced prostate cancer has scored major advances in the past few years. The OS in metastatic hormone-sensitive prostate cancer patients has been significantly prolonged upon androgen deprivation therapy combined with abiraterone acetate or docetaxel treatment [21] and the efficacy of these compounds will be soon tested in earlier stages of the disease. However, even in the light of these advances, recurrences will occur relatively fast due to the absence of immunogenic modulation which is essential for durable clinical efficacy. Immunotherapies-induced tumor-specific immune memory is an option for durable clinical responses. Yet, except for sipuleucel-T, single-agent immunotherapies in patients with prostate cancer have not demonstrated a clear progression-free survival or OS improvement in large clinical trials. It is therefore important to determine factors, including the clinical setting and patients' clinicopathological characteristics which could positively impact clinical responses to immunotherapies. Furthermore, by applying combination therapies and also selecting patients most likely to develop clinical responses to such therapies, we may overcome tumor evasion of the immune response. Given the low toxicity of therapeutic vaccines, investigating vaccination regimens in the setting of localized and biochemical recurrent prostate cancer may be advantageous. Immune checkpoint inhibitors are usually well tolerated and produce less toxicity when compared with cytotoxic chemotherapy, but, nevertheless, there are clinical 
cases to report an association of these agents with serious immune-related adverse events. PD-1/PD-L1 inhibitors have demonstrated improved tolerability over CTLA- 4 inhibitors, but caution should be exercised particularly with a patient population expected to have extended survival. Combining immunotherapy with chemotherapy is also an option for inducing antitumor activity and clinical efficacy, given that chemotherapy (e.g., docetaxel) by suppressing tumors and blocking immunosuppressive cell populations, may help overcome the tumor-induced immune tolerance. Hormonal therapies blocking either androgen production (abiraterone acetate) or signaling (enzalutamide) have demonstrated immunomodulatory capacities and combined with immunotherapies may also maximize clinical benefits. There are some questions that remain unaddressed with respect to other therapies administered with an immunotherapy: what will be the most effective chemotherapy or hormonal therapy? Which is the most effective combination to induce synergism? And what is the appropriate sequencing of application to foster maximum clinical benefit? And most important, can counteracting tumor promoting pathways and activating antitumor immunity lead to efficient immunoselection minimizing tumor heterogeneity with improved clinical outcomes? Current work on the discovery of prognostic and predictive biomarkers will certainly provide the clues for applying more effective therapeutic regimens in the near future.

\section{Conclusion}

Although there is an FDA-approved immunotherapy for mCRPC (sipuleucel-T), still prostate cancer is not considered as an immune-responsive tumor. Survival benefits from clinical studies with immune checkpoint inhibitors are limited and have yet to be maximized. For this, it will be essential to evaluate the effects of immunotherapies within the tumor microenvironment to be able to improve clinical results by counteracting acquired immune resistance induced during immune checkpoint therapy. Moreover, heterogeneity of neoantigen expression versus clonal neoantigen expression within the tumor microenvironment may also substantially influence the outcome of immunotherapies. Therefore, it will be essential to apply multiple treatment strategies in combination with immunotherapies to augment their potential. The upregulation within the tumor microenvironment of multiple regulatory immune checkpoints which could hinder any clinical responses also points to multiple immune checkpoint inhibition as an alternative option for combination therapies in prostate cancer. Although there is increasing tendency for combining various therapeutic modalities, the vast majority of studies in prostate cancer use single checkpoint inhibition with limited survival advantage, missing the opportunity for strong clinical benefit if multiple immune checkpoint inhibitors would be evaluated in sequence or in combination with other agents. Immunogenic modulation will provide the rationale for combinatorial strategies to be developed in future trials. Furthermore, appropriate patient selection based on predictive biomarkers and the mutational burden is also an option to improve the outcome of studies testing checkpoint inhibitors in prostate cancer. In the light of oncoimmunology trials in the various types of cancer, accumulating knowledge will allow for better design of clinical therapeutic protocols for prostate cancer to be applied in the appropriate clinical setting and potentially enhance clinical outcomes.

\section{Financial \& competing interests disclosure}

The authors have no relevant affiliations or financial involvement with any organization or entity with a financial interest in or financial conflict with the subject matter or materials discussed in the manuscript. This includes employment, consultancies, honoraria, stock ownership or options, expert testimony, grants or patents received or pending, or royalties.

No writing assistance was utilized in the production of this manuscript.

\section{References}

1. Baxevanis CN, Perez SA. Endogenous immunity at the forefront of tumor dormancy. Future Sci. OA 1(3), FSO13 (2015).

2. Becht E, Giraldo NA, Dieu-Nosjean MC, Sautes-Fridman C, Fridman WH. Cancer immune contexture and immunotherapy. Curr. Opin. Immunol. 39, 7-13 (2016).

3. Chowell D, Morris LGT, Grigg CM et al. Patient HLA class I genotype influences cancer response to checkpoint blockade immunotherapy. Science 359(6375), 582-587 (2018).

4. Riaz N, Havel JJ, Makarov V et al. Tumor and microenvironment evolution during immunotherapy with nivolumab. Cell 171(4), 934-949 e915 (2017).

5. Aptsiauri N, Ruiz-Cabello F, Garrido F. The transition from HLA-I positive to HLA-I negative primary tumors: the road to escape from T-cell responses. Curr. Opin. Immunol. 51, 123-132 (2018).

6. Campoli M, Ferrone S. HLA antigen changes in malignant cells: epigenetic mechanisms and biologic significance. Oncogene 27(45), 5869-5885 (2008). 
7. Sharma P, Hu-Lieskovan S, Wargo JA, Ribas A. Primary, adaptive, and acquired resistance to cancer immunotherapy. Cell 168(4), 707-723 (2017).

8. Snyder A, Makarov V, Merghoub T et al. Genetic basis for clinical response to CTLA-4 blockade in melanoma. N. Engl. J. Med. 371(23), 2189-2199 (2014).

9. Handy CE, Antonarakis ES. Sipuleucel-T for the treatment of prostate cancer: novel insights and future directions. Future Oncol. 14(10), 907-917 (2018).

10. Alaia C, Boccellino M, Zappavigna S et al. Ipilimumab for the treatment of metastatic prostate cancer. Expert Opin. Biol. Ther. 18(2), 205-213 (2018).

11. Gao J, Ward JF, Pettaway CA et al. VISTA is an inhibitory immune checkpoint that is increased after ipilimumab therapy in patients with prostate cancer. Nat. Med. 23(5), 551-555 (2017).

12. Benci JL, Xu B, Qiu $Y$ et al. Tumor interferon signaling regulates a multigenic resistance program to immune checkpoint blockade. Cell 167(6), 1540-1554 e1512 (2016).

13. Nuhn P, De Bono JS, Fizazi K et al. Update on Systemic prostate cancer therapies: management of metastatic castration-resistant prostate cancer in the era of precision oncology. Eur. Urol. doi:10.1016/j.eururo.2018.03.028 (2018) (Epub ahead of print).

14. Schumacher TN, Hacohen N. Neoantigens encoded in the cancer genome. Curr. Opin. Immunol. 41, 98-103 (2016).

15. Vogelstein B, Papadopoulos N, Velculescu VE, Zhou S, Diaz LA, Jr, Kinzler KW. Cancer genome landscapes. Science 339(6127), 1546-1558 (2013).

16. Boddupalli CS, Bar N, Kadaveru $\mathrm{K}$ et al. Interlesional diversity of $\mathrm{T}$ cell receptors in melanoma with immune checkpoints enriched in tissue-resident memory T cells. JCI Insight 1(21), e88955 (2016).

17. Jimenez-Sanchez A, Memon D, Pourpe $S$ et al. Heterogeneous tumor-immune microenvironments among differentially growing metastases in an ovarian cancer patient. Cell 170(5), 927-938 e920 (2017).

18. Isaacsson Velho P, Antonarakis ES. PD-1/PD-L1 pathway inhibitors in advanced prostate cancer. Expert Rev. Clin. Pharmacol. 11(5), 475-486 (2018).

19. Gevensleben H, Dietrich D, Golletz C et al. The immune checkpoint regulator PD-L1 is highly expressed in aggressive primary prostate cancer. Clin. Cancer Res. 22(8), 1969-1977 (2016).

20. Bishop JL, Sio A, Angeles A et al. PD-L1 is highly expressed in enzalutamide resistant prostate cancer. Oncotarget 6(1), 234-242 (2015).

21. Van Soest RJ, De Wit R. Irrefutable evidence for the use of docetaxel in newly diagnosed metastatic prostate cancer: results from the STAMPEDE and CHAARTED trials. BMC Med. 13, 304 (2015). 